\title{
Civilisations
}

Revue internationale d'anthropologie et de sciences

humaines

$54 \mid 2006$

Expériences de recherche en République

démocratique du Congo

\section{Enquête sur les perceptions du don bénévole de sang}

Positionnement et enjeu d'une recherche

\section{Gaëlle Fonteyne}

\section{(2) OpenEdition}

\section{Journals}

Édition électronique

URL : http://journals.openedition.org/civilisations/417

DOI : $10.4000 /$ civilisations. 417

ISSN : 2032-0442

Éditeur

Institut de sociologie de l'Université Libre de Bruxelles

Édition imprimée

Date de publication : 1 avril 2006

Pagination : 155-165

ISBN : 2-87263-006-6

ISSN : 0009-8140

\section{Référence électronique}

Gaëlle Fonteyne, «Enquête sur les perceptions du don bénévole de sang », Civilisations [En ligne], 54

2006, mis en ligne le 01 avril 2009, consulté le 10 décembre 2020. URL: http://

journals.openedition.org/civilisations/417 ; DOI : https://doi.org/10.4000/civilisations.417

(C) Tous droits réservés 


\title{
Enquête sur les perceptions du don bénévole de sang \\ Positionnement et enjeu d'une recherche
}

\author{
Gaëlle FONTEYNE
}

Résumé : Sur base d'une enquête socio-anthropologique menée à Kinshasa sur les obstacles et motivations au don de sang bénévole en RDC dans le cadre d'un programme de coopération au développement, cette contribution s'attache à illustrer une réflexion sur la place et le rôle d'une chercheuse vis-à-vis des populations qui participent à son étude. La première partie de cet article présente les méthodes de recherche privilégiées en fonction du cadre de travail auquel la chercheuse est confrontée; ensuite, les différentes étapes de cette enquête sont développées, ainsi que les difficultés et les solutions mises en æuvre. La deuxième partie se veut une réflexion critique sur la place et le rôle d'une chercheuse. Sur base de la façon de se présenter aux différents acteurs concernés et de leur propre perception du contexte, le statut de la chercheuse est constamment redéfini, renégocié en fonction des logiques et des stratégies de chaque acteur ou groupe d'acteurs impliqués dans la question de la promotion du don de sang bénévole.

Mots-clés : don de sang, santé publique, entretien, perception du chercheur, Kinshasa.

Summary: This article is based on a socio-anthropological study carried out in Kinshasa on the obstacles to and motivations for voluntary blood donations. The research took place in the framework of a development cooperation programme. The objective of this article is to reflect on the place and role of a researcher vis-à-vis the people participating in the study. The first part presents the research methods and the ways they are embedded in the work context. Next, the different steps of the study are outlined, along with obstacles and resolution strategies. The second part presents a critical reflection on the role of the researcher focussing on how she presents herself to actors involved in the project and how their perception of the context influences results. Experience proves that the status of the researcher has to be constantly redefined and renegotiated in relation to the logics and strategies of each actor or group of actors involved in the issue of promoting voluntary blood donations.

Key words: blood donation, public health, interview, perception of researcher, Kinshasa. 


\section{Introduction}

u début de l'année 2004, j'ai été engagée afin de mettre en oeuvre un projet de coopération au sein du Centre national de transfusion sanguine ${ }^{1}$ de la RDC; plus précisément, il s'agissait de fournir un appui technique à la cellule chargée de la promotion du don de sang bénévole en menant à bien une enquête socio-anthropologique sur le don de sang. Ce projet s'inscrit plus largement dans la lutte contre la transmission du VIH par voie sanguine ${ }^{2}$. Il a été prouvé que la sélection efficace et la fidélisation de donneurs de sang bénévoles et volontaires limitent le prélèvement d'unités de sang infectées et permet d'assurer une meilleure sécurité transfusionnelle pour le donneur et le receveur ${ }^{3}$.

\section{Contexte de la recherche}

L'objectif de cette enquête sur « Les obstacles et motivations au don de sang bénévole en RDC » était d'identifier et d'analyser les perceptions de la population par rapport au don de sang bénévole. Les résultats présentés ici portent essentiellement sur la première partie de l'enquête, soit des entretiens réalisés avec des donneurs de sang bénévoles ${ }^{4}$ issus des différentes communautés cibles identifiées (universités, écoles, Eglises, entreprises), principalement à Kinshasa et dans certaines provinces du pays (Katanga, Nord et Sud Kivu, Bas-Congo). Concrètement, j'ai travaillé plus d'une année au sein du CNTS, mais l'enquête proprement dite - observations, entretiens, analyse des résultats et rédaction s'est déroulée sur une période de quatre mois.

Pour résumer, il s'agit d'une recherche anthropologique commanditée par le Programme national de transfusion sanguine, un programme spécialisé du Ministère de la santé congolais, financée par un organisme de la coopération francophone belge, l'APEFE (Association pour la promotion de l'éducation et de la formation à l'étranger). Cette précision prend toute son importance dans le contexte particulier de la RDC et des relations entre l'Etat congolais, la Belgique et la population.

\section{Conditions matérielles d'enquête}

Affectée au CNTS, j'ai pu disposer d'un véhicule de l'administration publique ainsi que d'un chauffeur. J'ai donc pu faire le choix de rencontrer mes interlocuteurs dans leurs milieux respectifs (église, structure médicale, université, etc.) ce qui m'a permis de me rendre compte de leurs conditions de vie et de l'influence de celles-ci sur leurs représentations, leurs comportements. Les lieux où j'ai mené mes entretiens - un petit bureau sombre et étouffant, ouvert au passage et au bruit, un banc et un coin d'ombre au bout de la cour de l'église, etc. - ont nécessité à eux seuls une mobilisation importante de ma capacité d'adaptation. Quant au matériel, j'ai opté pour les « outils de base » : bic, carnet et enregistreur à piles (détail qui a son importance dans des quartiers où le délestage est la norme), bien adaptés à mes conditions de travail.

1. En sigle : CNTS. J'utiliserai cette abréviation dans la suite du texte.

2. La transmission du VIH par voie sanguine constitue le vecteur le plus " performant » c'est-à-dire qu'en présence d'une unité de sang infectée par le virus, le risque d'être contaminé est de plus de 90\%.

3. Outre la sélection de donneurs à faibles risques, le CNTS/Kinshasa teste systématiquement toutes les unités de sang pour le VIH, l'hépatite B et C, la syphilis, la malaria et les microfilaires.

4. La deuxième partie de l'enquête concerne les non-donneurs de sang ou les anciens donneurs. 


\section{Les méthodes de recherche}

Pour mener à bien cette étude, j'ai commencé par m'imprégner des réalités du terrain en circulant à travers les milieux, les groupes sociaux et auprès des différents acteurs impliqués :

- Au niveau du CNTS, suivi du travail de l'équipe chargée de la promotion du don, le personnel en charge de l'organisation et de la tenue des collectes, les médecins, les infirmiers, les laborantins; discussion autour de leur propre vision du don de sang.

- Au niveau des associations de donneurs bénévoles de sang, rencontres avec leurs membres et les responsables de la fédération des associations et, à de multiples reprises, échanges de point de vue sur la problématique des donneurs bénévoles.

- Au niveau des donneurs bénévoles et des donneurs potentiels de sang, participation aux prises de contacts, visites et séances de sensibilisation organisées dans les églises, les universités, les entreprises, etc.; attention particulière aux remarques et aux questions que le thème du don de sang suscite chez ces personnes.

- Enfin, accompagnement des équipes de prélèvement lors des collectes mobiles de sang afin d'observer les comportements et les interactions entre donneurs, membres des associations et personnel de collecte.

\section{L’imprégnation}

Mes premières semaines de présence sur place n'ont pas été très «actives », et je donnais probablement l'impression de me promener, de regarder partout et de poser beaucoup de questions : ce que Olivier de Sardan nomme «l'imprégnation » (2001 : 67), une période d'accoutumance au contexte social qui fut l'occasion pour moi d'être présentée à la majorité des acteurs impliqués dans la transfusion et le don de sang; non seulement, j'ai fait leur connaissance, et vice-versa, mais surtout, les raisons et la légitimité de ma présence ont été justifiées par les responsables du $\mathrm{CNTS}^{5}$. Cette accréditation a eu un impact important quoique difficile à évaluer sur mes relations avec les personnes rencontrées : certaines ont pu croire que j'étais chargée de les « espionner» pour le compte du CNTS, ou au contraire, que je pouvais jouer le rôle de relais.

\section{L'observation}

J'ai poursuivi le processus de cette recherche en menant de multiples observations des comportements et des relations qui s'établissent entre donneurs, personnel du CNTS et/ ou personnel chargé des collectes ${ }^{6}$ et membres des associations de donneurs. Toutes mes observations n'ont pas été scrupuleusement consignées dans mon carnet de bord, comme on nous apprend à le faire lors de notre formation initiale. Les méthodes d'observation doivent s'adapter au contexte et à la position dans laquelle la chercheuse se trouve : difficile

5. En effet, lors des premiers jours, j'étais toujours accompagnée par un membre du CNTS, souvent un de mes collègues de la cellule de promotion du don : il était le garant de ma présence et assurait ainsi ma légitimité auprès de mes interlocuteurs en m'introduisant auprès d'eux.

6. Les équipes chargées des collectes de sang sont constituées du personnel du CNTS et des autres banques de sang des hôpitaux de la ville faisant parties du réseau des banques de sang. 
pour la seule « blanche » du CNTS de s'asseoir tranquillement dans la salle d'attente sans susciter des regards, des questions, des commentaires et provoquer ainsi des interactions spontanées - mais pas toujours souhaitées - avec la population (même si parfois, cela a pu se révéler intéressant). J'ai donc dû mener mes observations au quotidien « sans en avoir l'air »; j'ai essayé de profiter des interactions auxquelles j'assistais (réunion, collecte, plate-forme, etc.) pour porter mon attention sur le type de relations entre les parties en présence. A ce titre, on peut réellement parler d'observation participante.

Et ce sont parfois de petits détails fortuits, saisis lors d'une interaction parmi d'autres qui m'apportaient un nouvel élément de réflexion : lors de la supervision d'une collecte dans une école, je discutais avec un des infirmiers chargés du prélèvement lorsque je remarquai qu'un donneur paraissait se sentir mal, il vacillait. Les autres personnes présentes avaient aussi leur attention attirée par ce donneur, elles le regardaient avec de l'inquiétude dans les yeux. L'un d'eux interpelle l'infirmier qui prend alors en charge ce donneur souffrant, sans fournir la moindre explication sur son état. C'est en discutant avec les donneurs présents que j'ai appris qu'ils ne recevaient aucune information sur les effets secondaires du don de sang (vertiges, maux de tête, évanouissement) et que devant de tels « accidents », ils prenaient peur et craignaient de se faire prélever, et ce d'autant plus que le personnel médical ne fournissait aucune explication pour rassurer les autres donneurs lorsque de tels incidents se produisaient.

\section{L'entretien}

Sur base de ces premiers éléments, j'ai élaboré un canevas d'enquête (cf. annexe) qui devait m'aider à «diriger» mes entretiens; ce canevas reprend d'abord quelques informations permettant d'identifier les personnes interviewées et d'établir un rapide et sommaire profil sociologique. Ensuite, sur base de mon appréhension générale de la situation, ainsi que d'après les problématiques et les questions soulevées par les différents acteurs rencontrés, mon " guide » tentait de saisir et de comprendre les perceptions du don de sang bénévole.

L'utilisation d'un guide d'entretien nécessite une grande souplesse : l'interlocuteur suit rarement l'ordre dans lequel les questions sont rédigées et certaines peuvent s'avérer inutiles ou mal formulées. Quoi qu'il en soit, il faut pouvoir s'en éloigner et saisir l'opportunité d'exploiter d'autres dimensions lorsque la personne interviewée vous en donne l'occasion, comme cet étudiant albinos qui m'expliquait les craintes de certains camarades lorsqu'il donnait son sang et leur refus de recevoir le sang d'un albinos, de peur de devenir albinos eux-mêmes. Or, dans mes précédents entretiens, la majorité des personnes acceptaient facilement l'idée de recevoir le sang d'un « blanc » puisque, selon eux, le sang est le même pour tous mais la question de l'albinos n'avait jamais été abordée précédemment.

Une fois mon canevas de recherche mis au point, j'ai commencé à mener des entretiens approfondis avec des donneurs bénévoles de sang d'abord. En accord avec le directeur du CNTS, Molipo, un bénévole bien intégré auprès des donneurs et des associations, m'a accompagné lors de mes rendez-vous afin de me présenter et d'être mon intermédiaire auprès des personnes que je rencontrais. Au départ, je lui ai laissé une certaine marge de manoeuvre dans le choix des sites et des interlocuteurs ${ }^{7}$, mais petit à petit, en fonction

7. Sur base du canevas que je lui donne et en fonction des discussions que nous avons à propos des objectifs de l'enquête. 
de la façon dont mon échantillon se constituait, j'ai orienté le choix du «profil » des personnes que je souhaitais rencontrer sur base des quelques rares statistiques disponibles sur la fréquentation du CNTS : une majorité d'hommes, et une moyenne d'âge de 30 ans. De plus, j'ai étendu mon échantillon aux différentes communautés à faible risque que le CNTS cible généralement : les universités et instituts supérieurs, les Eglises et les entreprises; et ce, afin de multiplier les points de vue sur le don.

A maintes reprises, j'ai discuté avec mon intermédiaire, Molipo, du contenu des entretiens : dans certains cas, il a pu les expliquer et/ou les compléter par un apport d'information. C'est pourquoi j'ai impliqué mon intermédiaire dans ma recherche afin qu'il puisse à son tour en saisir le sens et les implications. D'autant plus qu'à différentes reprises, ne maîtrisant pas la langue vernaculaire, j'ai dû faire appel à ses connaissances et à sa bonne volonté pour traduire certaines questions-réponses.

Au total, j'ai rencontré et interviewé 25 donneurs bénévoles de sang. La durée des entretiens à été variable, selon l'intérêt que la chercheuse et l'interlocuteur y trouvaient mais cela ne déterminait pas la qualité du contenu. J'ai mené des entretiens de deux heures qui se sont révélés peu utiles pour ma recherche alors que parfois, des informations importantes m'ont été communiquées sur une demi-heure d'interview. Dans ce domaine, il n'y a pas vraiment de normes et tout dépend de l'interaction qui s'établit entre les deux parties en présence.

Le travail le plus laborieux vint ensuite puisqu'il s'agissait d'écouter et éventuellement de transcrire ces entretiens. Bien que connaissant l'aspect fastidieux de ce travail, j'ai choisi de transcrire la plus grande partie de mes entretiens afin de me donner les moyens de réaliser un travail d'analyse et de comparaison approfondi.

Au quotidien, tout ce travail de recherche a également profité des nombreux échanges et conversations informelles que j'ai pu avoir avec les différents acteurs impliqués, ces échanges venant enrichir et compléter le recueil plus systématique des données. Si cette dimension particulière, intuitive, de la connaissance se révèle difficile à théoriser dans le cadre d'une recherche à visée scientifique, son importance me semble indéniable : beaucoup de choses peuvent se dire lors de ces échanges, plus intimes; de plus, ils participent à l'établissement de relations suivies et de confiance avec les personnes.

\section{L'analyse}

L'analyse des résultats d'une enquête constitue une des étapes les plus importantes et les plus enrichissantes, selon moi, du travail de recherche; mais c'est aussi l'étape la plus difficile à formaliser. Chaque anthropologue se constitue sa propre méthode au gré de ses expériences, de ses lectures, de ses rencontres. Je voudrais essayer ici d'en dire quelques mots, exposer la manière dont j'ai travaillé.

Comme je l'ai mentionné précédemment, j'ai retranscris presque intégralement tous les entretiens (cela n'est pas toujours possible et dépend du nombre, de la durée et de la qualité des enregistrements), ce qui m'a permis de les lire plusieurs fois attentivement et de repérer un certain nombre d'éléments de concordance et/ou de discordance dans le discours. J'ai également porté une attention particulière aux éléments qui me semblaient plus surprenants. Pour compléter mon analyse, j'ai dressé un tableau très simple mentionnant quelques informations de type sociologique afin d'établir d'éventuelles redondances dans un même groupe (les étudiants, les femmes, etc.) et ainsi mettre au jour des indicateurs pertinents. 
Petit à petit, à l'aide de fluorescent et de notes inscrites en marge de mes documents, des points d'analyse récurrents se sont dégagés (l'équilibre du sang, le sang comme principe vital, les liens du sang, etc.) qui, une fois organisés, ont formé le corps de mon analyse. Il me restait alors à m'atteler à la rédaction des résultats et de l'analyse de l'enquête proprement dits.

\section{Les questions soulevées : rôle et fonction de la chercheuse}

\section{Comment se présenter à son interlocuteur?}

Avant même d'engager le dialogue, certains éléments inhérents à ma personne parlaient pour moi. Mon apparence physique, une femme, jeune et blanche, me marquait déjà auprès de mes interlocuteurs et il est difficile de déterminer laquelle de ces caractéristiques a été la plus difficile à "gérer». Dans l'ensemble, je pense que mes interlocuteurs ont réagi positivement même si, à certaines occasions, mon âge ${ }^{8}$ et mon apparence les ont amenés à me considérer comme une étudiante : « et vous faites votre thèse sur quel sujet précisément? " « Vous venez de quelle université? » étaient des questions récurrentes.

De façon générale, j'ai toujours été bien accueillie par mes interlocuteurs qui ont tous accepté de répondre à mes interrogations. Aucune réticence, pas de demande de document officiel justifiant ma présence et mes activités, mais bien une curiosité légitime : « mais vous faites ça pour qui? A quoi ça va servir?».

Par ailleurs, j'ai toujours veillé à respecter les principes hiérarchiques en commençant par saluer le responsable du lieu où je me rendais (église, université, hôpital, etc.), lui expliquant le but de ma démarche et m'assurant de son autorisation, tout en n'oubliant pas de le remercier pour sa collaboration.

\section{Le statut de la chercheuse}

Cette recherche sur les obstacles et les motivations au don de sang s'inscrivait dans un cadre institutionnel bien particulier : j'étais une coopérante envoyée par la Belgique pour travailler au sein d'un Programme national congolais de santé publique et cela a suscité un certain nombres d'attentes de la part de mes interlocuteurs (et de ma part également). Ce n'est pas toujours évident pour une chercheuse d'arriver sous le couvert d'une institution étatique, surtout dans un pays comme la RDC où les relations Etat - population ne sont pas évidentes : «Mais quels rapports la société étudiée entretient-elle avec l'Etat? Et quelle position le chercheur étranger peut-il occuper à l'intérieur de ce rapport lorsqu'il est conflictuel? (...) Si l'Etat est son garant, ne risque-t-il pas d'apparaître comme un envoyé de l'Etat?» (Caratini 2004 : 44). Dans le cadre d'un oligopole comme Kinshasa, la perception de l'Etat et de ses représentants joue un rôle important dans l'établissement des relations avec la population.

Dans ce cas précis, ma « double appartenance » a pu s'avérer ambiguë : soit induire une certaine réserve dans le chef de certains de mes interlocuteurs qui cherchaient à contrôler la teneur des informations que l'on me donnait et qui s'inquiétaient de savoir quel usage sera fait de leurs propos; soit offrir une possibilité de contact et de relais, tant

8. Je précise que j'ai effectivement 25 ans au moment de l'enquête mais que généralement, les gens sousestiment mon âge. 
auprès du PNTS que de la coopération belge pour, par exemple, introduire une demande de projet :

(...) il y a un projet ici qu'on a élaboré (...), si on pouvait trouver des produits, des produits pharmaceutiques, quelques produits qui peuvent encore appuyer le laboratoire, si un donneur tombait malade, il peut facilement arriver ici chez nous et nos médecins consultent gratuitement et puis on l'envoie à la banque et donc, c'est ... c'est un petit projet peut-être qu'on va vous donner le lundi.

Ceci d'autant plus facilement qu'en tant que «blanche", mes interlocuteurs supposaient que ma crédibilité ne pouvait pas être mise en doute et que j'étais donc une intermédiaire fiable; c'est le concept d' « encliquage » selon lequel le chercheur se voit considéré comme le représentant ou le porte-parole du groupe qu'il étudie (Olivier de Sardan 2001 : 74). Ainsi, lors d'une série d'entretiens au sein d'une association de donneurs, alors que je m'apprêtais à clôturer la séance, le responsable de l'association s'est présenté pour l'entretien qu'il pensait avoir à propos de l'association en tant que telle. Quelle ne fut pas sa surprise lorsque je lui ai répondu que j'avais terminé et que je n'avais pas projeté de m'entretenir avec lui, sauf s'il souhaitait me parler de son expérience de donneur!

Ma démarche, en ce qu'elle s'intéressait également aux difficultés rencontrées par les donneurs représentait aussi une opportunité, pour mes interlocuteurs, de faire entendre leur voix :

(...) la semaine passée, il y a notre camarade qui est décédé à la clinique Bondeko et sa famille n'avait pas d'argent. Il fallait que nous nous débrouillions pour avoir de l'argent pour le corps de notre ami. Alors, on nous a dit que si on voulait avoir le corps, il fallait que trois personnes cèdent le sang. Si on donnait du sang, ils nous rendaient le corps de notre ami sans que l'on doive donner de l'argent. Ce n'est pas facile mais il y en a deux qui ont cédé leur sang...

ou encore, de profiter de la rencontre interpersonnelle pour poser des questions sur le don de sang :

(...) je ne sais pas si c'est vrai ou c'est faux mais c'est quelque chose qui se dit ici à Kinshasa: quand vous prenez la grenadine, c'est-à-dire que vous voyez, la grenadine, c'est pour donner plus de sang. Moi, je prends la grenadine quand je suis malade, mais sinon, je prends la bière brune; (...) semble-t-il quand on prend de la bière brune, ça donne beaucoup de sang, je sais pas?

Plus simplement, certains interlocuteurs me demandaient mes coordonnées et venaient au CNTS pour me rendre visite et me présenter à d'autres de leurs amis donneurs.

\section{Le discours}

Est-ce que mon statut d'expatriée travaillant pour la coopération belge, affectée à un Programme spécialisé du Ministère de la santé congolais a eu une influence sur le discours de mes interlocuteurs? En fait, quel que soit le statut de la chercheuse, dans l'interaction avec les personnes interviewées, le contenu du discours des uns et des autres s'adapte en fonction des intérêts et des attentes de chacun. J'ai observé plusieurs types de réactions liées à mes différentes « caractéristiques » :

En tant qu'étrangère, certains sujets sont difficiles à aborder. Les thèmes de la sorcellerie et de la religion constituent des exemples classiques. Le don de sang touche 
également à l'utilisation du sang dans certains rituels magico-religieux, tels que les pactes de sang. Mes interlocuteurs n'ont pas refusé d'en parler mais leurs réponses semblaient standardisées et assez vagues;

(...) c'est une pratique qui est en voie de disparition : au moins dans les milieux ruraux, ça existe encore (...). Ce sont des pratiques qu'on condamne : certaines personnes ont peur d'être liées ... vous savez, en Afrique, il y a beaucoup de mythes sur le sang,

comme pour éviter d'approfondir le sujet et le rejeter comme de vielles pratiques dépassées.

En tant que représentante du CNTS, mes interlocuteurs hésitaient à émettre des remarques ou des critiques vis-à-vis du personnel et de leur travail. Non pas que je cherchais à provoquer une réaction négative, mais lors de mes observations, il était manifeste qu'il y avait des manquements (mauvais accueil, manque de respect, ...) et certains donneurs ne se gênaient pas pour manifester ouvertement et parfois bruyamment leur mécontentement lors des collectes. Mais durant les entretiens interpersonnels, aucun n'a formulé la moindre remarque négative à l'encontre du CNTS; parlant du personnel du CNTS, « ils sont gentils », voilà ce qui est ressorti de la majorité des entretiens.

Enfin, en tant que coopérante belge, certains de mes interlocuteurs, principalement les responsables des associations de donneurs bénévoles de sang, ont été amenés à me présenter une image ambivalente de la situation du don :

On a beaucoup de donneurs, au moins 500 inscrits et on trouve des nouveaux sites encore. Et un peu plus loin : (...) si vous pouvez penser à nous parce que il y a des associations de donneurs qui n'ont même pas les moyens, si vous pouvez penser à eux et envoyer des habits, à manger.

D'une part, ils mettaient en avant l'importance de leurs activités auprès des donneurs (recrutement, sensibilisation, motivation, suivi, etc.) en me présentant les résultats positifs; d'autre part, ils insistaient beaucoup sur leurs difficultés à trouver de nouveaux donneurs, les petits moyens avec lesquels ils étaient obligés de travailler, le manque de personnel, les problèmes de déplacement, etc.

Dans certains cas, un tiers ${ }^{9}$, assistant à l'entretien, intervenait dans le discours de mon interlocuteur afin de rectifier un détail, d'apporter des données supplémentaires ou d'insister sur un point; lors d'une séance d'entretien avec un ouvrier de la brasserie Bracongo alors que je l'interrogeais sur la remise des résultats des tests sanguins, il m'expliquait que les résultats avaient été affichés à l'infirmerie (alors que cela doit rester anonyme) : c'est à ce moment que le responsable de l'association des donneurs de la Bracongo est intervenu pour m'expliquer que ce n'étaient pas les résultats en tant que tels qui étaient communiqués mais bien le nombre de personnes qui avaient des résultats positifs. Voulant approfondir la question avec la personne que j'interviewais, le responsable de l'association répondait systématiquement à sa place pour m'expliquer les modalités décidées par l'association quant à la question de la remise des résultats. Impossible d'obtenir l'avis de l'ouvrier en question.

9. Cela peut être mon intermédiaire, le responsable de l'association dont fait partie le donneur interviewé, ou encore le pasteur de l'Eglise dont j'occupe momentanément le bureau. 


\section{Conclusion}

L'objectif premier de cette enquête était d'identifier les motivations et les obstacles au don bénévole de sang afin d'adapter les stratégies de promotion du don au contexte de la RDC. A terme, ce projet de coopération a pour objectif d'augmenter le nombre de donneurs bénévoles de sang, qui présentent le moins de risque de transmettre des maladies, jusqu'à attendre $100 \%$ de don bénévole pour couvrir l'entièreté des besoins du pays.

Si l'on en revient à la question centrale de l'apport des sciences sociales à la santé publique, il faut maintenant se demander quelle peut être l'utilité d'une telle analyse pour un décideur ou un acteur du système de santé. Plus précisément, quelles sont les chances que cette connaissance critique (...) influe effectivement sur le cours des décisions et des actions de santé publique? (Fassin $1994: 6$ )

Les résultats de l'enquête sont disponibles mais se pose encore tout le problème de la publication et de l'utilisation de ces données. A ce jour, le constat est mitigé, il faut maintenant entreprendre un autre type de travail afin concrétiser l'apport réel de ces données dans le travail quotidien de la promotion du don de sang bénévole.

Mais au-delà de cet objectif initial, d'autres enjeux sont apparus : pour bien comprendre ce que représentait cette recherche et ma présence auprès des acteurs impliqués dans la promotion du don de sang bénévole, il faut savoir que le PNTS est un programme d'envergure qui bénéficie de l'apport de nombreux bailleurs de fonds importants tels que la Banque Mondiale, la coopération allemande, CDC/Atlanta, ONUSIDA, CORDAID, la CTB, l'APEFE, etc., ce qui explique que, comparativement aux autres structures publiques de santé, l'infrastructure, la logistique et les conditions de travail sont très satisfaisantes. Ainsi, outre les données de l'enquête, ce qui apparaît au détour des entretiens menés, c'est aussi toute la question du financement et de la rémunération des activités liées à la transfusion, entre autres la promotion du don bénévole, puisque les associations y prennent une part active et qu'en retour, elles essayent d'y gagner quelques avantages, matériels, financiers ou symboliques. Pour les donneurs aussi, l'enjeu était de taille, cela représentait l'occasion de se faire entendre, de revendiquer leurs droits (bénéficier de soins de santé gratuits) ou de manifester leur mécontentement (ne pas vendre le sang qu'ils donnent bénévolement ${ }^{10}$ ).

10. Le CNTS cède les poches de sang au prix de 10USD, conformément à la politique de recouvrement des coûts; mais en fait, le prix réel de « fabrication » d'une poche de sang prélevée et testée est approximativement de 50USD (coût du matériel, salaire du personnel, carburant, amortissement du matériel, intrants, etc.). C'est un problème réel vis-à-vis des donneurs bénévoles qui estiment que le CNTS gagne de l'argent sur leur dos. 


\section{Références bibliographiques}

Caratini, Sophie, 2004. Les non-dits de l'anthropologie. Paris : PUF.

Fassin, Didier, 1994. "L'anthropologue et la santé publique», in Anthropologie, santé, maladie : autour d'études de cas, numéro spécial du Bulletin de l'AMADES. Accessible à l'adresse : http://www.amades. net/publications/txtligne/txt04/fassin.pdf

Olivier DE SARDAN, Jean-Pierre, 2001. « L'enquête de terrain socio-anthropologique », in J. Boutier, J.-L. Fabiani et Jean-Pierre Olivier de Sardan (éds), Corpus, sources et archives. Actes des journées d'études de l'Institut de recherche sur le Maghreb contemporain, pp. 63-111. Tunis : Ed. IRMC.

\section{Annexe : Enquête sur la perception, les motivations et les obstacles au don bénévole de sang dans la population de Kinshasa : guide d'entretien}

Les donneurs de sang bénévoles :

Identification :

- nom, prénom, post-nom

- sexe

- âge

- profession

- formation

- religion

- famille

- membre d'une association de donneurs

- histoire personnelle

- a déjà donné du sang pour un membre de la famille, un proche

- autre(s) membre(s) de la famille qui donne(nt) aussi du sang

- avez-vous déjà eu besoin d'une transfusion, ou un membre de votre famille?

Le sang :

- perception du sang

- d'où vient le sang?

- connaissances (quels types de connaissances, d'où viennent-elles?)

- histoire personnelle, souvenirs à propos du sang

- sang et bien-être

- sang et santé

- concept de transmission par le sang (maladie mais aussi foi, religion, caractère, etc.)

- infections transmissibles

- sang blanc/sang noir

- lien du sang

- sang des animaux

- la peur du sang

- que peut-on faire avec du sang, à quoi cela sert?

- le sang peut-il faire du mal?

Le don :

- premier don - impressions

- contexte du don 
- pourquoi ?; avantages, intérêt du don, motivation

- quels sont les arguments qui ont convaincu?

- bénéficiaire - savoir pour qui on donne

- accompagnement lors du don

- comment on se sent après le don?

- la piqûre

- combien de temps cela prend?

- l'annonce des résultats

- connaissances et comportements à risque

- l'exclusion ou l'auto-exclusion

- faire partie d'un groupe différent

Le centre de transfusion :

- accueil

- appréciation du personnel

- appréciation du centre

- informations connues sur le don de sang

- la collation

- information reçue sur le don, les comportements à risque, etc.

- attitude du personnel : avant, pendant et après le don

- la façon dont le personnel s'occupe de vous

- la façon dont le personnel vous considère

- comment se déroule votre parcours lorsque vous arrivez pour un don ? 
